\section{Valore del farmaco, innovazione e criteri di valutazione}

\section{Dear Editor,}

L'articolo sul tema dell'innovazione, elaborato da un gruppo di esperti che operano presso la Regione Veneto e pubblicato nel presente numero di Global \& Regional Health Technology Assessment (2015; 2(2): 107-113) è il prodotto di un interessante confronto multidisciplinare tra i clinici più autorevoli della Regione, promosso dal Servizio Farmaceutico della Regione Veneto e dal Gruppo Aziende Europee Farma.

Dopo una prima fase dedicata al brain storming, alla discussione e alla selezione dei principali elementi - sia teorici che operativi - da dibattere, l'esperienza si è sviluppata per trovare un "consenso" tra i diversi stakeholder del sistema su alcuni elementi critici del processo di Health Technology Assessment (HTA).

Questo Position Paper, seppure prodotto in un contesto regionale specifico, costituisce una fotografia efficace dell'attuale livello di evoluzione del dibattito italiano in materia di HTA in generale, e di innovazione in particolare.

Con questa chiave di lettura, numerosi sono i commenti che questo dibattito e questo Position Paper suscitano.

In primo luogo, se ne sentiva la mancanza: in altri Paesi (in primis il Regno Unito) da tempo sono maturate esperienze molto simili; in Italia, questa iniziativa della Regione Veneto - pur limitata nel tempo e nelle risorse investite - colma una sorta di vuoto lasciato dalle istituzioni nel confronto multidisciplinare tra stakeholder portatori di prospettive diverse.

In secondo luogo, questa esperienza rappresenta al tempo stesso il punto di arrivo e il punto di partenza per un percorso, oramai ineludibile, che intende esplicitare e rendere (più) trasparenti i principi base e le regole decisionali nei processi di valutazione.

È un punto di arrivo perché questo Position Paper testimonia il compimento di un necessario processo di condivisionetra clinici, decisori e industria che fa ricerca - dei concettibase che governano la materia [ad esempio: (a) adottare la sopravvivenza quale indicatore di esito clinico per valutare l'efficacia clinica dei nuovi farmaci ad alto costo e per proporzionare le risorse economiche destinate ai singoli farmaci sulla base dei benefici clinici aggiuntivi; (b) quando la sopravvivenza da sola non descrive adeguatamente il beneficio del trattamento, privilegiare l'uso del QALY per la sua quantificazione]. È, al tempo stesso, un punto di partenza perché già da adesso si profilano all'orizzonte ulteriori interrogativi, più specialistici e talora più raffinati, ma anch'essi ormai ineludibili.

Tra le nuove questioni che emergono in prospettiva futura, molte risultano collegate e proposte dai nuovi farmaci anti-HCV, il cui percorso decisionale è necessariamente più complesso e metodologicamente più sofisticato rispetto ai percorsi che abbiamo sperimentato storicamente. In questo quadro, può essere utile esaminare, con un certo dettaglio, alcune delle "nuove" questioni emerse.

(1) Con sofosbuvir, il beneficio si realizza dopo circa 15-20 anni dalla somministrazione della terapia, il che impone secondo la teoria economica di adottare un correttivo metodologico robusto che riduca il valore del beneficio quando quest'ultimo si colloca lontano nel tempo; questo correttivo è il tasso di sconto sui benefici (solitamente tra 1,5-3\% per anno), che ad esempio il NICE usa fin dalla sua nascita nel 1998; essendo un "parametro specialistico", entrare nei dettagli della sua determinazione può sembrare una forzatura, ma dato l'impatto sui risultati sarebbe una forzatura anche non farne cenno.

(2) Quando l'impatto finanziario è molto grande (es. NAO o ranibizumab) o addirittura enorme (sofosbuvir), è pratica comune per le istituzioni (o i decisori) quella di sottoscrivere con l'azienda produttrice un accordo prezzo/volume; il problema è che non esistono linee prestabilite di comportamento in questo campo, cosicché la quantificazione economica di questa relazione resta discrezionale e/o priva di regole. II tutto si aggrava perché questi accordi, sia in Italia che all'estero, vengono gestiti in totale confidenzialità; questa permette sconti maggiori ma evidentemente rende poco trasparente il processo: si tratta quindi di una questione indubbiamente molto complessa. A questo proposito alcuni parlamentari italiani hanno recentemente sollevato questo specifico problema proponendo, come soluzione, quella di una trasparenza solo italiana (evidentemente non di facile realizzazione). Le esigenze di trasparenza su questo punto sono innegabili, ma - a nostro avviso servirebbe soprattutto portare avanti una richiesta di trasparenza a livello europeo, piuttosto che a livello dei singoli Stati.

(3) Mentre in passato il nostro SSN ha potuto rimborsare la (quasi) totalità dei farmaci approvati da EMA, con i nuovi farmaci anti-HCV si pone, per la prima volta in Italia, la questione del "dover dire No"; su questo versante, I'Italia non ha purtroppo maturato un suo "vissuto" che abbia coinvolto gli stakeholder (istituzioni, clinici, pazienti, cittadini) nella condivisione della scelta delle priorità: la questione si pone adesso per la prima volta e all'improvviso (1). I decisori sembrano orientati a lasciar fuori dalla rimborsabilità una notevole quota-parte di pazienti con HCV che invece potrebbero beneficiare dei nuovi farmaci, e a mantenere all'interno della rimborsabilità tutti i trattamenti oncologici accettandone l'elevato prezzo anche nei casi in cui il beneficio sia quantitativamente molto piccolo (pur se statisticamente significativo). Si tratta di questioni molto rilevanti e impegnative, su cui il dibattito, in casa nostra, è solo agli albori e comunque stenta a decollare.

(4) Il mercato farmaceutico è completamente diverso da quello di soltanto 10 anni fa, sia per le diverse caratteristiche delle molecole innovative, sia per un crescente tasso di concorrenza creato da bioequivalenti e biosimi- 
lari; i mercati potenziali delle nuove molecole sono sempre più piccoli e la loro obsolescenza sempre più rapida: da questo punto di vista i farmaci tendono a incorporare problematiche e caratteristiche che storicamente sono sempre state attribuite al settore dei dispositivi medici; la decrescente capacità di protezione dei brevetti e la spinta alla crescita dei prezzi sono quindi fattori ineludibili, che richiederanno un ripensamento globale della governance del settore, che non può rimanere ancorato a logiche a silos come quelle che sinora hanno governato il SSN.

\section{Disclosures}

Financial support: No financial support was received for this submission.

Conflict of interest: The authors have no conflict of interest.

\section{Andrea Messori ${ }^{1}$, Federico Spandonaro ${ }^{2}$}

\footnotetext{
${ }^{1}$ Responsabile HTA, ESTAR (Ente di Supporto Tecnico Amministrativo Regionale) - Regione Toscana, Firenze - Italy

${ }^{2}$ Università degli Studi di Roma Tor Vergata Presidente CREA Sanità, Roma - Italy
}

Indirizzo per la corrispondenza:

Andrea Messori

Responsabile HTA, ESTAR (Ente di Supporto Tecnico Amministrativo Regionale) - Regione Toscana, Firenze, Italy

andrea.messori.it@gmail.com

\section{Bibliografia}

1. Steinbrook R. Saying no isn't NICE - the travails of Britain's National Institute for Health and Clinical Excellence. N Engl J Med. 2008;359(19):1977-81.

Published online: May 13, 2015 